\title{
Ein Hymnus auf die Lichtjungfrau
}

\author{
Jessica Kristionat / Gregor Wurst (Augsburg)
}

Im Kontext eines seit mehreren Jahren laufenden Forschungsprojektes ${ }^{1}$ zur Gruppe der manichäischen Sonntagspsalmen (Nr. 119-130) sowie der beiden auf sie folgenden Psalmengruppen (Nr. 131-135 und 136-149) aus dem ersten Teil des manichäischen Psalmenbuches ${ }^{2}$ ist auch das Verhältnis des Psalmes Nr. 134 aus Teil I zu dem von C.R.C. Allberry als „Psalmus ad animam victricem “ publizierten Text zu behandeln. ${ }^{3}$ Wie schon aus den Abschriften in der unpublizierten Work Concordance to the Coptic Manichaean Psalmbook (2002) von W.P. FunK hervorgeht, handelt es sich bei diesen beiden Psalmen um eine Dublette. ${ }^{4}$ Damit liegt nicht nur ein weiterer Beleg für eine doppelte Textüberlieferung einzelner Psalmen sowohl innerhalb als auch außerhalb des Psalmenbuches vor, ${ }^{5}$ sondern der Befund erlaubt auch wesentliche Korrekturen an den Lesungen Allberrys und seiner Interpretation des Textes als eines an die anima victrix gerichteten Psalmes. Im Folgenden legen wir zunächst die Editionen der beiden Texte vor, ${ }^{6}$ woran ein kurzer exegetischer Kommentar anschließt.

1 Das Projekt wurde in den Jahren 2001-2004 durch die Deutsche Forschungsgemeinschaft (im Rahmen der Gewährung eines Heisenbergstipendiums an G. WURST) und in den Jahren 2008-2010 durch die Universität Augsburg durch eine wissenschaftliche Mitarbeiterstelle für J. Kristionat gefördert. Beiden Institutionen sind die Verfasser zu großem Dank verpflichtet.

2 Vgl. FacEd III Taf. 161-208; der Titel der Sonntagspsalmen ebd. Taf. 161, Z. 8 ab imo; die Titel der beiden folgenden Gruppen sind unbekannt, da sie vom Schreiber des Codex im Text nicht notiert worden sind (vgl. die Taf. 182 und 189) und der Index am Ende des zweiten Teils des Psalmenbuches nur unleserliche Spuren bietet (vgl. Psb. 231,10. 16). - Zur Gruppe der Sonntagspsalmen vgl. bislang Wurst 1997/1998; Wurst 1999.

3 Vgl. FacEd III Taf.187-188; Psb. 118,1-30.

4 Dies hat Funk in seiner Work Concordance zwar nicht explizit festgestellt, seine vom Text Allberrys divergierende Abschrift von Psb. 118 zeigt jedoch, dass er sich dieses Befundes bewußt war.

5 Vgl. dazu Wurst 1995, S. 47-51 sowie Gardner 1996, Ss. 43-49. 55. 67-72.

6 Die Editionen wurden auf der Grundlage neuer digitaler Photographien des Blattes FacEd III Taf. 187-188 sowie anhand von FacEd IV Taf. 118 erarbeitet. Die Abschriften FunKs (Work Concordance, S. 1071f.; 1132), die neben der Edition Allberrys auf FacEd $I I I$ und $I V$ beruhen, wurden dabei dankbar verwendet, ohne jedoch jede Divergenz in der Lesung zu notieren. 


\section{Edition von Psb. 118,1-30 und Psalm Nr. 134}

Die Seiten 117 und 118 des zweiten Teils des Psalmenbuches weisen sowohl in der Edition Allberrys als auch in der Faksimile-Edition einen stark differierenden Erhaltungszustand auf, obwohl es sich um Vorder- und Rückseite ein und desselben Blattes handelt. ${ }^{7}$ Dieser Befund erklärt sich vor dem Hintergrund einer von S. GIVERSEN publizierten Notiz unklarer Herkunft, die folgendermaßen lautet: „In foliis seperandis (A. D. MDCCCCXXXV) pars paginae periit. Superest quod vides. "8 Das bedeutet also, daß Allberry den Text der Vorderseite (p. 117) wohl auf der Grundlage eines Photos bzw. einer Autopsie vor Ablösung des Blattes vom Buchblock gelesen hat; bei seiner Ablösung wurde es dann aber offensichtlich stark beschädigt, so daß es zu massivem Textverlust kam und das Blatt heute nur noch in der in der Faksimile-Edition dokumentierten Form erhalten ist.

Darüber hinaus ist bekannt, daß sich der Erhaltungszustand des Codex seit den Tagen AllberRYs generell verschlechtert hat, so daß auch seine Lesungen der p. 118 heute nicht mehr vollständig nachzuvollziehen sind. Entsprechend den Vorgaben der Neu- bzw. Erstedition des Psalmenbuches im Rahmen des Corpus Fontium Manichaeorum ${ }^{9}$ werden von Allberry eindeutig bezeugte Lesungen übernommen. Zwei offensichtliche Fehler, die ihm bei seiner Abschrift unterlaufen sind, sind im Apparat jedoch verzeichnet.

Wie der Vergleich der beiden Textzeugen zeigt, hat Allberry den Textverlust am Anfang von p. 118 mit nur einer Zeile zu gering eingestuft. Seine erste Textzeile Psb. 118,3 - Psb. 118,2 enthält wohl nur die Notierung des Refrains - entspricht im Paralleltext FacEd III Taf. 187 der Zeile 23; die unleserliche Zeile Psb. 118,1 wird also FacEd III Taf. 187 Zeile 22 entsprechen, so daß davor noch zwei Zeilen für den Text von FacEd III Taf. 187 Zeilen 20-21 anzusetzen sind..$^{10}$ Diese beiden anzunehmenden Zeilen werden in der folgenden Edition als p. 118,a-b gezählt. Darüber hinaus ergeben sich aus dem Vergleich der beiden Textzeugen mehrere Änderungen in der Lesung oder bei Textergänzungen von Psb. 118,2-15, die im Wesentlichen von Funk schon gesehen wurden und aufgrund ihrer Evidenz nicht näher kommentiert werden müssen. P. 119, die

7 Vgl. Psb. 117 / FacEd IV Taf. 117 mit Psb. 118 / FacEd IV Taf. 118.

8 FacEd IV Taf. 117 Anm.

9 Vgl. Wurst 1996, S. 13-14.

10 Der numerierte und im Index erfasste Teil des Psalmenbuches endet mit Psb. 113,23, so dass hier auf der Seite Psb. 118 nicht noch eine weitere Zeile mit der Psalmenzahl zu rekonstruieren ist. 
das Ende des Psalmes (parallel FacEd III Taf. 188,6-8) enthielt, ist vollständig unleserlich. ${ }^{11}$

Das Blatt FacEd III Taf. 187-188 mit dem Text des Psalmes 134 ist fast vollständig erhalten und gut lesbar, zeigt jedoch an mehreren Stellen den auch im zweiten Teil des Psalmenbuches nachgewiesenen Befund, daß sowohl auf seiner Vorder- als auch auf seiner Rückseite kleinere oder größere Fragmente des vorangehenden oder nachfolgenden Blattes kleben, die im Zuge der Konservierung nicht abgelöst wurden. ${ }^{12}$ So werden auf Taf. 187,20-25 die Initialen der Zeilen/ Verse durch ein längliches Fragment des vorangehenden Blattes großenteils verdeckt, ebenso auf Taf. 187,20-21 die Zeilenenden - der in der Edition in eckige Klammern gesetzte koptische Text ist an diesen Stellen also nicht zerstört, sondern durch Papyrusfragmente abgedeckt. Dasselbe gilt für das Zeilenende von Taf. 187,30 sowie für die Zeilenanfänge von Taf. 188,1-8. Weder die auf Taf. 187,19 rechts neben der Psalmenzahl in der Faksimile-Edition sichtbaren Buchstaben noch jene auf Taf. 188 links von der Psalmenzahl (am Zeilenanfang) des folgenden Psalmes gehören zu diesem Blatt.

Psb. 118,1-30

$118, \mathrm{a}$

tres lineae perierunt

1

NIC)

3

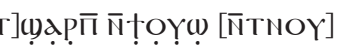

[N] $\mathrm{E}$ NI(I)

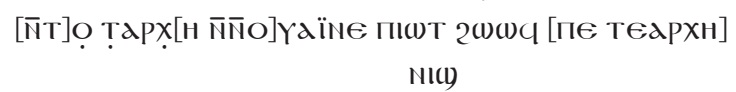

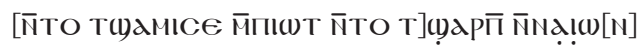

$\mathrm{N}$ [IC)]

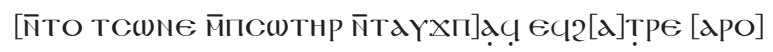

NI(1)

17

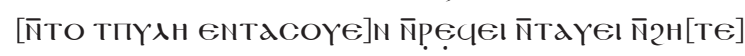

NI(1)

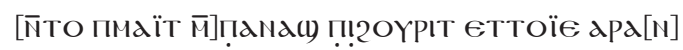

NI(1)

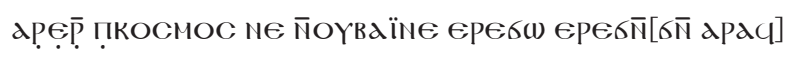

NI(1)

11 Vgl. Psb. 118 Anm.

12 Vgl. Wurst 1996, S. 11-12. 


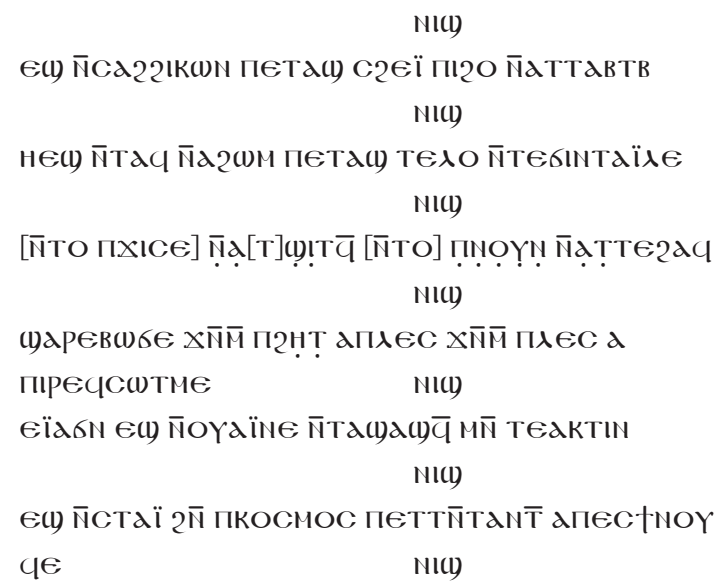

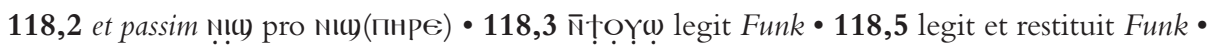

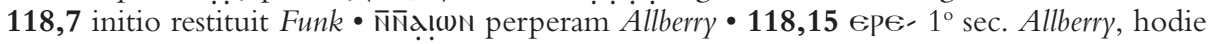

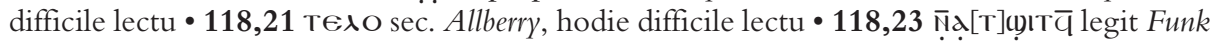
- 118,26 міресс(1)тме perperam Allberry

Psb. 118,1-30

$$
\begin{array}{ll}
118, \mathrm{a} & {[---]} \\
\mathrm{b} & {[---]} \\
1 & {[---]}
\end{array}
$$

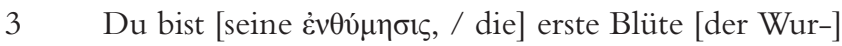

$4 \quad$ [ze]l. die $W$ (under)

5 [D]u bist der Anfan[g der] Lichter, / der Vater selbst [ist dein Anfang.] die $W$ (under)

$7 \quad$ [Du bist die Erstgeborene des Vaters, / du bist die] Erste der Äonen. die $W$ (under)

9 [Du bist die Schwester des Erlösers / der erschaffen wurde] und sich [mit dir] die $W$ (under) vereinigt hat.

11 [Du bist das Tor, das sich geöffnet hat,] / durch [dich] sind die Kommenden

$$
\text { die } W \text { (under) }
$$
gekommen.

13 [Du bist der Weg] des Schwures, / der Wächter, der [uns] vorsteht.

$14 \quad$ die $W$ (under)

15 Du hast dir den Kosmos zu einer Harfe gemacht, / unaufhörlich spielst du [auf ihm.] 
die $W$ (under)

$17 \quad[---]$

Welcher Ikonenmaler ist es, / der dies ungeschmückte Gesicht wird malen die $W$ (under) die $W$ (under)

21 Oder welcher Adler ist es, / der deinen Aufstieg wird ersteigen können ? die $W$ (under)

23 [Du bist die] unermessliche [Höhe, / du bist] die unerreichbare Tiefe. die $W$ (under)

Du springst vom Herzen auf die Zunge, / von der Zunge auf

$$
\text { den Hörer. die W(under) }
$$

Welches Licht werde ich finden, / auf dass ich es mit deinem Lichtstrahl vergleiche?

die $W$ (under)

Was für ein Duft ist in der Welt, / der deinem Wohlgeruch gleicht? die $W$ (under)

FacEd III Taf. 187,18-188,8

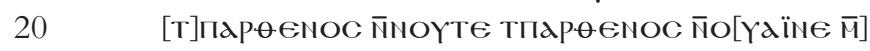

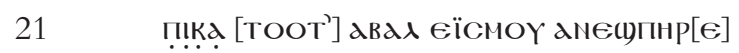

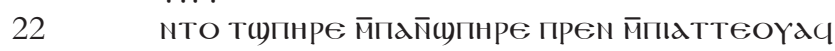

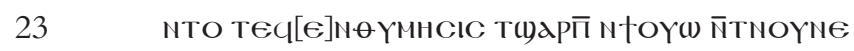

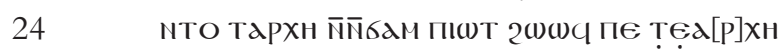

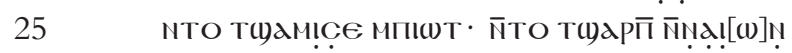

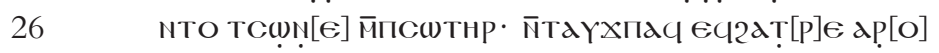

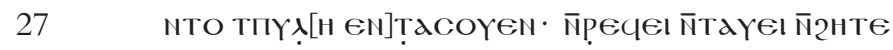

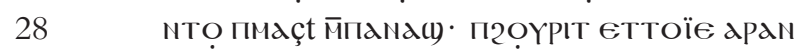

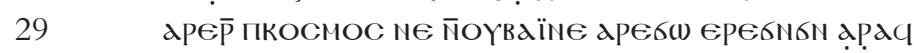

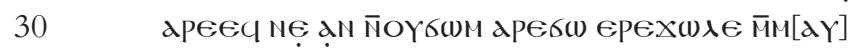

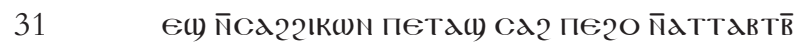

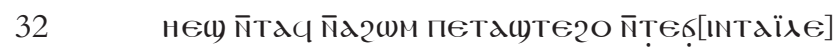

188,1 [нто пхісє]

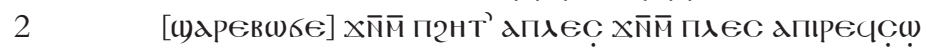

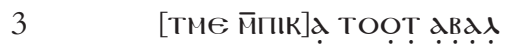

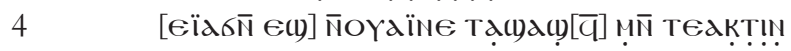

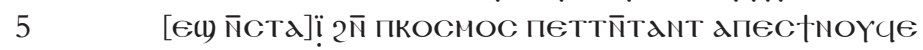

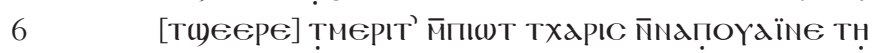




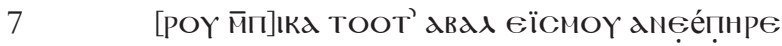

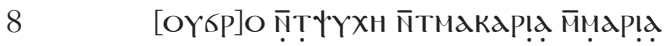

187,22-28 nTO vestigiae supralineationum super litteris initialibus non supersunt $\bullet \mathbf{1 8 7 , 2 4}$

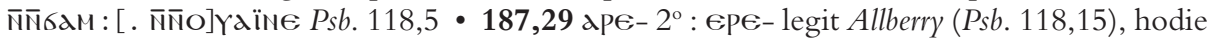
difficile lectu • 187,31 ca2 пє2о : с2єї пıго Psb. 118,19 • 187,32 тє2О : тє৯о leg. Allberry (Psb. 118,21), hodie difficile lectu • 188,4 Td-: ̄̄Td-Psb. 118,27

FacEd III Taf. 187,18-188,8

187,19

134

20 (Du) göttliche Jungfrau, / (du) [Licht]jungfrau!

21 Ich habe nicht aufgehört, / deine Wunder zu preisen.

22 Du bist das Wunder des Wunderbaren, / der Name des Unsagbaren.

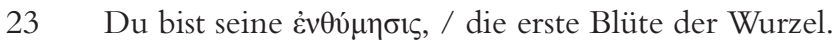

24 Du bist der Anfang der Mächte, / der Vater selbst ist dein Anfang.

25 Du bist die Erstgeborene des Vaters, / du bist die Erste der Äonen.

26 Du bist die Schwester des Erlösers, / der erschaffen wurde und sich mit dir vereinigt hat.

27 Du bist das Tor, das sich geöffnet hat, / durch dich sind die Kommenden

28 Du bist der Weg des Schwures, / der Wächter, der uns vorsteht.

29 Du hast dir den Kosmos zu einer Harfe gemacht, / unaufhörlich hast du auf ihm gespielt.

30 Du hast ihn dir auch zu einem Garten gemacht, / unaufhörlich hast du $\mathrm{d}[$ ort $]$ geerntet.

31 Welcher Ikonenmaler ist es, / der dein ungeschaffenes Gesicht wird malen können?

32 Oder welcher Adler ist es, / der deinen [Aufstieg] wird erreichen können?

188,1 [Du bist die] unermessliche [Höhe], / [du bist] die unerreichbare Tiefe.

2 [Du springst] vom Herzen auf die Zunge, / (und) von der Zunge auf den

3 Hörer. [Ich habe] nicht aufgehört.

4 [Welches] Licht [werde ich finden], / auf dass ich es mit deinem Lichtstrahl vergleiche?

5 [Was für ein Duft] ist in der Welt, / der deinem Wohlgeruch gleicht?

$6 \quad$ [(Du) Tochter,] Geliebte des Vaters, / (du) Gnade a[1l] derer, die zum Licht

7 gehören. [Ich habe] nicht aufgehört deine Wunder zu preisen.

8 [Sie]g der Seele der seligen Maria. 


\section{Kommentar}

Der Vergleich der beiden Textzeugen beweist, dass der Text dieses Hymnus sehr einheitlich überliefert ist. Nur wenige Textvarianten sind festzustellen:

\begin{tabular}{|c|c|c|c|c|}
\hline \multicolumn{3}{|c|}{ Psb. 118} & \multicolumn{2}{|c|}{ FacEd III Taf. 187-188 } \\
\hline a) & 118,5 & 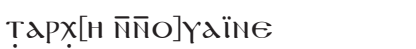 & 187,24 & Тархн ज̄N̄бдM \\
\hline b) & 118,15 & 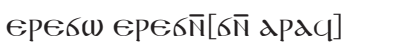 & 187,29 & 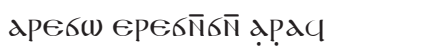 \\
\hline c) & 118,19 & пєТд() с2єї пі2О & 187,31 & П૯ТА(1) Сд2 Пєе2О \\
\hline d) & 118,21 & 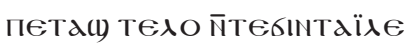 & 187,32 & 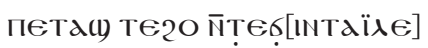 \\
\hline e) & 118,27 & $\overline{\text { NT}}$ Tdyde $\overline{\mathrm{q}}$ & 188,4 & $\operatorname{Td}(y)(y[\bar{q}]$ \\
\hline
\end{tabular}

Im Fall der Varianten b) und c) ist aus Gründen des Parallelismus Ursprünglichkeit für den Text von FacEd III anzunehmen: vgl. das vierfache ap€- in Taf.

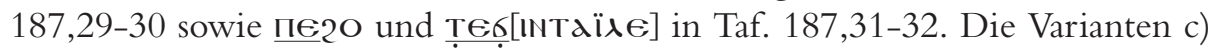
$\mathrm{C}_{2} € \mathrm{i}-$ / Cd2- und e) $\mathrm{NTd}-/ \mathrm{Td}$ - als unterschiedliche Formen des status nominalis sowie der ersten Person sing. des Konjunktivs liegen innerhalb der Variationsbreite des Dialektes der koptischen Manichaica, sind also wenig aussagekräftig. ${ }^{13}$ Schwieriger ist es jedoch, die Varianten a) und d) zu beurteilen: Ist im ersten Fall eine Entscheidung über die Ursprünglichkeit unmöglich, da die beiden Lesarten gleichwertig sind, mag im zweiten Fall das Stilmittel der figura etymologica (т€лO - бintä̈ле / „ersteigen“ - „Aufstieg“) die Priorität der Lesart von Psb. 118 nahelegen. Sicher ist dies jedoch keineswegs, da Allberrys eindeutige Lesung des Verbums TEגo heute so nicht mehr nachzuvollziehen ist.

Was die Gattung betrifft, beginnt der Psalm mit einer direkten Anrede der Lichtjungfrau (FacEd III Taf. 187,20), deren „Wunder zu preisen“ nicht aufgehört zu haben, der Psalmist sich rühmt (Taf. 187,20-21). Diese zweite Aussage ist mit dem Refrain zu identifizieren, der nach jedem Distichon wiederholt wird. ${ }^{14}$ In FacEd III Taf. 187f ist der Refrain zwar nur dreimal notiert ${ }^{15}$ in Psb. 118 hingegen nach jedem Vers, wenn auch in einer extrem verkürzten Form. Der Psalm erweist sich somit von vorn herein als ein Hymnus, und entsprechend besteht der Text durchgängig aus Prädikationen entweder im einfachen („,Du

13 Für c2єї- / cd2-vgl. Hom. 81,24 und Keph. 7,31; für ñTd- / Td-vgl. z. B. Psb. 108,2728.

14 Die hier vorgelegten Editionen geben die beiden Textzeugen zwar diplomatisch wieder, die Verstruktur des Psalmes ist in den Übersetzungen jedoch durch den Schrägstrich angedeutet. Der Refrain ist kursiv gesetzt.

15 FacEd III Taf. 187,20-21; 188,3. 7. 
hast ...“ etc.) oder im anaphorischen „Du“-Stil (wiederholtes „Du bist ...“ am Versanfang)..$^{16}$

Die Lichtjungfrau ${ }^{17}$ (тпарөємос N̄oүä̈re) gehört zu den Emanationen der dritten Berufung: Nach Keph. 35,7-17 bringt der aus dem Vater der GröBe emanierte Dritte Gesandte seinerseits die Säule der Herrlichkeit, Jesus den Glanz und die Lichtjungfrau hervor. Sie wird mit der (ursprünglich gnostischen) Weisheit $(\Sigma o \varphi i ́ \alpha)$ in Verbindung gebracht ${ }^{18}$ und als Gegnerin der Archonten angesprochen: „... die Lichtjungfrau, die herrliche Weisheit, die das Herz der Archonten und der Kräfte wegnimmt durch ihre Erscheinung, indem sie den Willen der Größe erfüllt. “19 In der Zeit der Vermischung ${ }^{20}$ tritt die Lichtjungfrau somit im Kontext der Erlösung des Lichts auf, dessen Läuterung bereits mit der zweiten Berufung durch den Vater der Größe begonnen hat. Darüber hinaus gilt sie auch als eine Emanation Jesu des Glanzes (Hom. 86,10-11; Psb. 2,27-29).

Die Position der Lichtjungfrau im manichäischen System ist jedoch ambivalent, wie ihre Austauschbarkeit mit den ,zwölf Jungfrauen“ zeigt. ${ }^{21}$ Ihre Hauptfunktion im manichäischen Mythos bildet die Szene der sogenannten „Verführung der Archonten", in der sie aufgrund ihres wandelbaren Geschlechts den männlichen und weiblichen Archonten der Finsternis die Lichtsubstanz entlockt. ${ }^{22}$ Obwohl auf diese ihre wichtigste Funktion nicht angespielt wird, korrespondiert die Darstellung der Lichtjungfrau in diesem Psalm im Wesentlichen mit jener im siebten Kapitel der Kephalaia: Ist sie hier die „Schwester des Erlösers" (Psb. 118,9-10 // FacEd III Taf. 187,26), so wird sie dort als zur selben Berufungsgeneration wie Jesus der Glanz gehörig bezeichnet, was ein geschwisterliches Verhältnis impliziert (Keph. 35,9-17). Dazu stehen die Prädikationen der Lichtjungfrau als ,,erste Blüte der Wurzel“, „Anfang der Äonen/[L]ichter“ oder „Erstgeborene des Vaters“ etc., mit denen sie im Psalm 134 in eine direkte Beziehung zum Vater der Größe gesetzt wird (niemand anders ist mit der „Wurzel“ gemeint, vgl. Psb. 134,15: „Die [Wurzel] ist der Gott der Wahrheit“; Keph. 35,3: „Dies ist der erste Vater, ... die Wurzel aller Lichter ...") nicht im Widerspruch. Nach dem von J.P. Asmussen formulierten ,manichäischen Prinzip der Identität" handelt in allen Berufungen/Emanationen des manichäischen Pan-

16 Vgl. Wurst 1995, S. 91-92.

17 Vgl. zuletzt Panaino 2011.

18 Keph. 24,18-19; 44,8-9; 172,20-24.

19 Keph. 35,14-17.

20 Zu den „drei Zeiten“ des manichäischen Mythos (Urzeit, Zeit der Vermischung/Mittlere Zeit und Endzeit), vgl. Keph. 55,17-57,32 sowie NAgel 1974, S. 201-214 und Wurst 1994, S. 167-179.

21 Vgl. Keph. 25,20-22.

22 Vgl. Colpe 1981, S. 60-62; Polotsky 1935, S. 257; Schmidt/Polotsky i933, S. 68. 
theons letztlich immer nur der „Vater“ selbst, ${ }^{23}$ entsprechend können sie auch in unterschiedliche Beziehungen zu ihm gesetzt werden. So wird in Psb. 137,22-23 die ,Jungfrau des Lichts“ auch mit einem der Söhne des Urmenschen, dem Element des „lebendigen Feuers“, und in Keph. 84,19-20 sogar mit der „Seele des Vaters" identifiziert.

Die übrigen Prädikationen der Lichtjungfrau in diesem Psalm stehen überwiegend in einem soteriologischen Kontext:

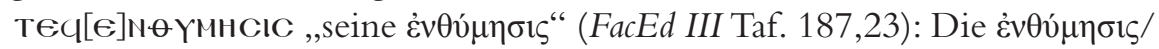
,Überlegung` ist die vierte der fünf Verstandeskräfte, mit denen das Wesen des Vaters der Größe charakterisiert wird; vgl. z. B. Keph. 64,20-24.

пүגн „Tor“ (FacEd III Taf. 187,27): Zum Motiv des geöffneten Tores vgl. Psb. 62,25-26; 156,1-8; 169,5-6.

пмаїт м̄пана(1) „Weg des Schwures“ (Psb. 118,13 // FacEd III Taf. 187,28): Diese etwas sperrige Formulierung, zu der es in den koptischen Manichaica keine weitere Parallele gibt, ist wohl dahingehend zu verstehen, dass das Wort „Schwur“, also eine ,heilige Verpflichtung', hier metaphorisch für das Bekenntnis eines Manichäers zu seinem Glauben steht. Der „Weg des Schwures“ ist also nichts anderes als der Weg des manichäischen Glaubens, über dessen Bekenner die Lichtjungfrau als „Wächter“ eingesetzt sei, wie es im zweiten Halbvers dieses Distichons heißt.

вдїnє „Harfe“ (Psb. 118,15 // FacEd III Taf. 187,29): Die Musik hatte bei den Manichäern einen hohen Stellenwert, ${ }^{24}$ nach dem Zeugnis Augustins wurde ihr ein göttlicher Ursprung zugeschrieben. ${ }^{25}$ Der himmlische, dem Vater der Größe dargebrachte Gottesdienst wird im Psalmenbuch häufig erwähnt: vgl. z. B. Psb. 133,18-22; 199,16-19; 200,5-9. Das Distichon preist die Lichtjungfrau also als diejenige, die die liturgische Verehrung des Vaters der Größe in dieser Welt bewirkt.

б(1) „Garten“ (FacEd III Taf. 187,30): Das Motiv vom Garten, das häufig in manichäischen Texten verwendet wird $^{26}$, beschreibt entweder den endzeitlichen Zustand des Paradiesgartens, in den aufgenommen zu werden, Ziel der Gläubigen ist (Psb. 8,14-21; Psb. 224,17-22), oder in Variation mit den Motiven

23 Vgl. Asmussen 1965, S. 12.

24 Vgl. z. B. Psb. 155,8; 164,9-10. 18-19; zu Musik und Hymnologie bei den Manichäern allgemein vgl. Puech 1979, S. 179-233.

25 Aug., mor. 2,46: Ita dulcedo musica, quam de divinis regnis venisse contenditis, nobis mortuarum carnium sordibus exhibetur ... „Diese Süße in der Musik, die, wie ihr behauptet, aus den göttlichen Reichen stammt, wird uns vom Schmutz toten Fleisches dargebracht ..." (Rutzenhöfer 204-205); vgl. Puech 1979, S. 188: „La liturgie terrestre est une réplique de la liturgie céleste."

26 Zum Motiv des Gartens allgemein vgl. Oerter 1991, S. 263-272. 
des Feldes bzw. Ackers ,den Ort des Wirkens der Lichtboten und Apostel“. ${ }^{27}$ Wie der Bauer vom Feld erntet die Lichtjungfrau täglich das Licht im Garten des Kosmos - bzw. ,,von ihm“, da FacEd III Taf. 187,30 am Zeilenende auch zu $\bar{M} M[\alpha C]$ ergänzt werden könnte.

a2wм „Adler“ (Psb. 118,21 // FacEd III Taf. 187,32): Auch das Motiv des Adlers verweist in den soteriologischen Bereich und begegnet auch im Kontext des Seelenaufstiegs, vgl. Psb. 64,18-19; 100,29-31. ${ }^{28}$

\section{Abkürzungen}

Aug., mor. = Elke Rutzenhöfer: Augustinus. De moribus ecclesiae catholicae et de moribus manichaeorum. Die Lebensführung der katholischen Kirche und die Lebensführung der Manichäer. Zweisprachige Ausgabe, Paderborn et al. 2004 (Augustinus, Opera, Werke T. 1: Bd. 25).

FacEd III = Søren Giversen: The Manichaean Coptic Papyri in the Chester Beatty Library III. Psalm Book Part 1. Facsimile Edition, Genève 1988 (Cahiers d'Orientalisme 16).

FacEd IV = Ders.: The Manichaean Coptic Papyri in the Chester Beatty Library IV. Psalm Book Part 2. Facsimile Edition, Genève 1988 (Cahiers d'Orientalisme 17).

Hom. = Hans Jаков Polotsky: Manichäische Homilien, Stuttgart 1934 (Manichäische Handschriften der Sammlung A. Chester Beatty 1).

Keph. = Alexander Böhlig / Hans Jakob Polotsky: Kephalaia. Erste Hälfte. Lieferung 1-10, Stuttgart 1940 (Manichäische Handschriften der Staatlichen Museen Berlin 1).

Psb. = Charles R.C. Allberry: A Manichaean Psalmbook. Part 2, Stuttgart 1938 (Manichaean Manuscripts in the Chester Beatty Collection 2).

27 Nagel 1980, S. 107-109 mit zahlreichen Beispielen (Zitat: ebd. S. 108); vgl. ebd. 122$124 \mathrm{zu}$ Thom(as)-Psalm Nr. 18.

28 Für eine Interpretation von Psb. 100,10-33 vgl. Richter 1997, S. 144-149. 


\section{Bibliographie}

Asmussen, Jes Peter (1965): Xuāstvānīft. Studies in Manichaeism, Copenhagen (Acta Theologica Danica 12).

Colpe, Carsten (1981): Daēnā, Lichtjungfrau, Zweite Gestalt. Verbindungen und Unterschiede zwischen zarathustrischer und manichäischer Selbst-Anschauung. In: Gilles Quispel / Roelof van den Broek / Maarten J. Vermaseren (Hrsg.), Studies in Gnosticism and Hellenistic Religions presented to Gilles Quispel on the occasion of his 65th birthday, Leiden (Études préliminaires aux religions orientales dans l'empire romain 91), S. 58-77.

Gardner, Iain (1996): Kellis Literary Texts 1. Oxford, (Dakhleh Oasis Project: Monograph 4).

Nagel, Peter (1974): Bemerkungen zum manichäischen Zeit- und Geschichtsverständnis. In: Ders. (Hrsg.), Studia Coptica, Berlin (Berliner byzantinistische Arbeiten 45), S. 201-214.

Nagel, Peter (1980): Die Thomaspsalmen des koptisch-manichäischen Psalmenbuches übersetzt und erläutert, Berlin (Quellen, Neue Folge 1).

Oerter, Wolf B. (1991): Das Motiv vom Garten. Betrachtungen zur manichäischen Eschatologie. In: Alois van Tongerloo / Søren Giversen (Hrsg.), Manichaica Selecta. Studies presented to Professor Julien Ries on the Occasion of his Seventieth Birthday, Leiden (Manichaean Studies 1), S. 263-272.

Panaino, Antonio (2011): Sadwēs, Anāhīd and the Manichaean Maiden of Light. In: Zekine Özertural / Jens Wilkens (Hrsg.), Der östliche Manichäismus. Gattungsund Werksgeschichte. Vorträge des Göttinger Symposiums vom 4./5. März 2010, Berlin (Abhandlungen der Akademie der Wissenschaften zu Göttingen, Neue Folge 17. Schriften der Kommission „Manichäische Studien“ 1), S. 121-131.

Polotsky, Hans J. (1935): Manichäismus. In: Pauly-Wissowa, Realenzyclopädie der classischen Altertumswissenschaft, Suppl. 6, S. 240-271.

Puech, Henri-Charles (1979): Musique et hymnologie manichéennes. In: Ders., Sur le Manichéisme et autres essais, Paris, S. 179-233.

Richter, Siegfried (1997). Die Aufstiegspsalmen des Herakleides. Untersuchungen zum Seelenaufstieg und zur Seelenmesse bei den Manichäern. Wiesbaden (Sprachen und Kulturen des christlichen Orients 1).

Schmidt, Carl / Hans Jakob. Polotsky (1933): Ein Mani-Fund in Ägypten. Originalschriften des Mani und seiner Schüler. Mit einem Beitrag von H. IBscher. In: SPAW, Berlin, S. 4-90 mit 2 Tafeln.

Wurst, Gregor (1994): Zur Bedeutung der 'Drei-Zeiten'-Formel in den koptisch- manichäischen Texten von Medînet Mâdi. In: Andreas Kessler / Thomas Ricklin / Gregor Wurst (Hrsg.), Peregrina Curiositas. Eine Reise durch den orbis antiquus. Zu Ehren von Dirk Van Damme, Freiburg (Schweiz) (Novum Testamentum et Orbis Antiquus 27), S. 167-179.

Wurst, Gregor (1995): Das Bêmafest der ägyptischen Manichäer, Altenberge (Arbeiten zum spätantiken und koptischen Ägypten 8). 
Wurst, Gregor (1996): The Manichaean Coptic Papyri in the Chester Beatty Library. Psalm Book, Part II, Fasc. 1. Die Bema-Psalmen, Turnhout (Corpus Fontium Manichaeorum. Series Coptica, I, Liber Psalmorum, Pars II, Fasc. 1).

Wurst, Gregor (1997/1998): Conférence [Gnose et Manichéisme]. In: Annuaire de l'École Pratique des Hautes Études, Section sciences religieuses 106, S. 333-334

Wurst, Gregor (1999): Die Bedeutung der manichäischen Sonntagsfeier (Manichäisches Psalmenbuch, I, 127). In: Stephen Emmel / u.a. (Hrsg.): Ägypten und Nubien in spätantiker und christlicher Zeit. Akten des 6. Internationalen Koptologenkongresses Münster, 20.-26. Juli 1996. Bd. II. Schrifttum, Sprache und Gedankenwelt, Wiesbaden (Sprachen und Kulturen des christlichen Orients 6,2), S. 563-580. 